\title{
SINGLE CENTER EXPERIENCE IN AWAKE THORACOSCOPIC CHEST SURGERY
}

\author{
Sami Kaan COSARCAN ***, Alper Tunga DOGAN*, Omur ERCELEN**, \\ Mete MANICI***, Suat ERUS**** \\ Department of Anesthesiology, Amerikan Hospital Istanbul Turkey \\ *DESA-Anesthesiology Specialist, **Professor, ${ }^{* * *}$ Anesthesiology Specialist, **** Thoracic Surgeon
}

\section{Background}

Video-assisted thoracoscopic surgeries contribute positively to postoperative complications, length of hospital stay and management of pain. We aimed to present our experience in awake thoracoscopic surgeries with high thoracic epidural to the patients with limited functional lung capacity.

\section{Cases}

8 patients underwent uniportal thoracoscopy and thoracoscopic surgery. Epidural catheter was inserted at the T3-4 level in the operation room. $2 \mathrm{mg}$ of morphine and $25 \mathrm{mg}$ of Bupivacaine were administered from the epidural catheter. T2 - L1 sensory block formation was observed in patients.

Pleurectomy -pleurodesis was performed in 7 and wedge resection in one of 8 patients. The mean operation time was $25.4 \mathrm{~min}$, and in 2 patients operation was 45 min. Two patients had coughing during the operation, stellate ganglion block was performed, and the surgery proceed. None of the patients developed intraoperative complications. No hemodynamic changes were observed.

\section{Results}

Before the end of the surgeries non-steroid anti-inflammatory drug and paracetamol was given. Opioids not needed. None of the patients complained about the pain. No complication was recorded.

\section{Conclusion}

We believe that thoracic epidural, and awake surgery are safe and usable in patients with poor general condition and comorbidities. Two important conditions that can be seen in awake thoracic surgery are coughing during the operation and postoperative period of open pneumothorax. The complaint of cough that may occur is disturbing both the patient's comfort and the surgical team, and the stellate ganglion block is highly beneficial in our case. Awake thoracoscopy also begins to take place in Enhanced Recovery After Surgery protocols (ERAS) which are tried to be formed in thoracic surgery anesthesia. We believe awake thoracoscopic surgery can safely performed in selected patients. 